\title{
AFAP-110 is overexpressed in prostate cancer and contributes to tumorigenic growth by regulating focal contacts
}

\author{
Jing Zhang, ${ }^{1,2}$ Serk In Park, ${ }^{1,2}$ Marlene C. Artime, ${ }^{1,2}$ Justin M. Summy, ${ }^{1}$ Ami N. Shah,1,3 \\ Joshua A. Bomser, ${ }^{4}$ Andrea Dorfleutner, ${ }^{5}$ Daniel C. Flynn, ${ }^{5}$ and Gary E. Gallick, \\ ${ }^{1}$ Department of Cancer Biology, The University of Texas MD Anderson Cancer Center, Houston, Texas, USA. 2Program in Cancer Biology, \\ The University of Texas Graduate School of Biomedical Sciences, Houston, Texas, USA. ${ }^{3}$ Department of Surgical Oncology, The University of Texas \\ MD Anderson Cancer Center, Houston, Texas, USA. ${ }^{4}$ Department of Human Nutrition, The Ohio State University, Columbus, Ohio, USA. ${ }^{5}$ Mary Babb Randolph \\ Cancer Center and Department of Microbiology, Immunology, and Cell Biology, West Virginia University, Morgantown, West Virginia, USA.
}

\begin{abstract}
The actin filament-associated protein AFAP-110 is an actin cross-linking protein first identified as a substrate of the viral oncogene v-Src. AFAP-110 regulates actin cytoskeleton integrity but also functions as an adaptor protein that affects crosstalk between Src and PKC. Here we investigated the roles of AFAP-110 in the tumorigenic process of prostate carcinoma. Using immunohistochemistry of human tissue arrays, we found that AFAP-110 was absent or expressed at very low levels in normal prostatic epithelium and benign prostatic hyperplasia but significantly increased in prostate carcinomas. The level of AFAP-110 in carcinomas correlated with the Gleason scores. Downregulation of AFAP-110 in PC3 prostate cancer cells inhibited cell proliferation in vitro and tumorigenicity and growth in orthotopic nude mouse models. Furthermore, downmodulation of AFAP-110 resulted in decreased cell-matrix adhesion and cell migration, defective focal adhesions, and reduced integrin $\beta 1$ expression. Reintroduction of avian AFAP-110 or a mutant disabling its interaction with Src restored these properties. However, expression of an AFAP-110 lacking the PKC-interacting domain failed to restore properties of parental cells. Thus, increased expression of AFAP-110 is associated with progressive stages of prostate cancer and is critical for tumorigenic growth, in part by regulating focal contacts in a PKC-dependent mechanism.
\end{abstract}

\section{Introduction}

The actin filament-associated protein of $110 \mathrm{kDa}$, termed AFAP-110, was first discovered in the early 1990s as one of several major substrates of the viral oncogenic protein tyrosine kinase v-Src $(1,2)$. Subsequent work has demonstrated that AFAP-110 functions as an actin filament cross-linking protein that has a fundamental role in actin cytoskeleton arrangement (3). AFAP-110 contains a carboxyterminal actin-binding domain and directly binds to F-actin (4). AFAP-110 undergoes self-multimerization through its leucine zipper domain, which allows the formation of a complex with multiple actin-binding sites to cross-link actin filaments. Depending on its concentration and phosphorylation status, AFAP-110 is able to organize actin filaments to form either a loose meshwork or tight bundle structures (5).

AFAP-110 also interacts with multiple binding partners through its several functional domains, including 2 pleckstrin homology (PH) domains, a Src homology 3-binding (SH3-binding) motif, and several SH2-binding motifs (6). AFAP-110 binds to PKC via its amino-terminal PH domain and is phosphorylated by this serine/ threonine kinase. PKC phosphorylation upregulates the ability of AFAP-110 to cross-link actin filaments, possibly by reducing AFAP110 self-association (7). The SH2- and SH3-binding motifs are involved in the association of AFAP-110 with the activated form of

Nonstandard abbreviations used: AFAP-110, actin filament-associated protein of $110 \mathrm{kDa}$; BPH, benign prostatic hyperplasia; CI, confidence interval; FAK, focal adhesion kinase; $\mathrm{PH}$, pleckstrin homology; $\mathrm{SH} 3$, Src homology 3.

Conflict of interest: The authors have declared that no conflict of interest exists. Citation for this article: J. Clin. Invest. 117:2962-2973 (2007). doi:10.1172/JCI30710.
Src. A site-directed proline to alanine mutation at the SH3-binding motif abrogates this interaction (8). Both amino- and carboxyterminal PH domains may potentially direct the association of AFAP110 to WD40 repeat-containing proteins, such as the receptor of activated protein kinase $\mathrm{C}$ (RACK1), as well as phospholipids at the cellular membrane (6). Thus, AFAP-110 functions as an adaptor protein that brings signaling molecules to specialized signaling complexes and/or subcellular compartments, affecting the location and crosstalk between these molecules. As a result, AFAP-110 may be important in regulating multiple cellular processes involving formation and dissolution of actin-based structures.

Disruption of actin organization abrogates the formation of focal adhesion structures and disturbs integrin-mediated signaling cascades $(9,10)$. Thus, adaptor proteins that relay enzymatic signals to actin filament networks are essential participants in cell-ECM interactions in both normal and malignant cells. Indeed, previous studies have identified many of the actin cytoskeleton-associated proteins as major effectors in oncogene-mediated alterations of cell morphology, cytoskeletal organization, and adhesive interactions. Further, emerging evidence demonstrates the multifunctional roles of actin-binding proteins in tumorigenic and metastatic processes of various human tumors. For example, cortactin, an actin-bundling protein, is frequently overexpressed and considered a prognostic marker in several cancers, such as breast cancer and squamous cell carcinoma of the head and neck (11-13). Downregulation of cortactin expression by siRNA impairs cancer cell motility and invasion (14). Cortactin overexpression inhibits ligand-mediated downregulation of EGF receptor by affecting the interaction of this receptor with c-Cbl ubiquitin ligase (15). Therefore, actin cytoskel- 


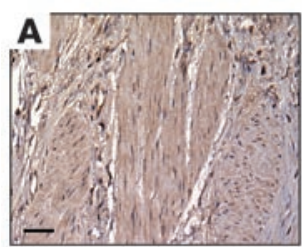

Smooth muscle

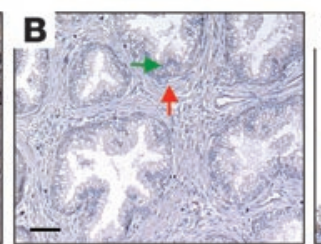

Normal epithelium

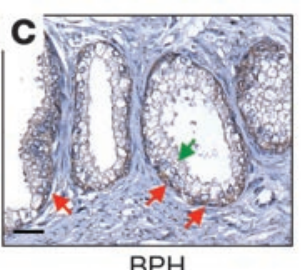

$\mathrm{BPH}$

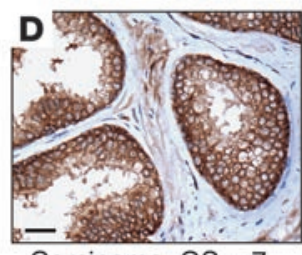

Carcinoma, GS $<7$

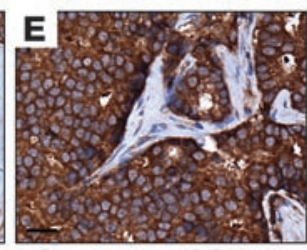

Carcinoma, GS $\geq 7$

\section{Figure 1}

Expression of AFAP-110 in normal and pathologic prostate tissues. Human prostate tissue arrays were subjected to immunohistochemical analyses with a monoclonal mouse anti-AFAP-110 primary antibody and a biotinylated goat anti-mouse secondary antibody after antigen retrieval. Brown indicates positive staining. Representative images from tissues with different pathologic characteristics are shown. GS, Gleason score. Scale bars: $100 \mu \mathrm{m}$. (A) Prostatic smooth muscles. Note that AFAP-110 is expressed in the cytoplasm of smooth muscle cells. (B) Normal prostate epithelium. Green arrow indicates luminal secretory cells, and red arrow indicates basal layer cells. (C) BPH. Note that immunoreactivity for AFAP-110 is not present in the secretory epithelial cells (green arrows). The proliferating basal cells (red arrows) demonstrate weak to moderate staining. (D) Prostatic adenocarcinoma with a Gleason score less than 7. (E) Prostate adenocarcinoma with a Gleason score greater than or equal to 7. Note that tumor foci demonstrate strong labeling for AFAP-110, while connective tissues in the stroma of prostate cancer specimen are not stained for AFAP-110.

eton-associated proteins that modulate both actin filament organization and intracellular signal transduction are not only targets of oncogenic proteins, they also directly contribute to tumor development and progression when their expression is altered.

Several lines of evidence from cell culture studies indicate a potential role of AFAP-110 in tumorigenic processes. First, transfection of a mutant form of AFAP-110, which lacks the leucine zipper domain, into fibroblasts induces a morphological change similar to that of $\mathrm{v}$-Src-transformed cells, including disruption of cytoplasmic stress fibers and increased podosome formation, suggesting a potential function of AFAP-110 in malignant transformation. Second, AFAP-110 has been shown to be important in the formation of specific actin-based structures known to be involved in malignant migration and invasion, such as PKC $\alpha$ induced podosomes $(4,16)$. Third, AFAP-110 has an intrinsic ability to activate Src, and this ability is regulated by PKC phosphorylation $(3,16)$. When aberrantly expressed or activated, both Src and PKC propagate a wide range of intracellular signal pathways deregulating cancer cell adhesion, migration, proliferation, invasion, and apoptosis $(17,18)$. Therefore, alterations in the expression of AFAP-110 that affect actin cytoskeleton integrity and intracellular signaling cascades might be expected to contribute to tumor growth and metastasis, although no current studies directly demonstrate AFAP-110 regulation of these processes.

Here we investigated the expression and functional roles of AFAP110 in human prostate carcinoma, one of the most frequent cancers in men in the United States and one in which aberrant PKC and Src signaling have been documented in the regulation of malignant progression (19-21). Using a prostate tissue array, we demonstrate that AFAP-110 is overexpressed in nearly every prostate carcinoma. Additionally, the levels of AFAP-110 positively correlated with the aggressiveness of the disease. Reduction of AFAP-110 in prostate cancer cells by stable siRNA expression suppressed cell proliferation in vitro, as well as tumor formation and growth in orthotopic nude mouse models. Downregulation of AFAP-110 also led to disrupted focal adhesion structures and induced a decrease in integrin $\beta 1$ expression. These effects required the functional domain that is essential for the association of AFAP-110 with PKC but not with Src. Collectively, our data show that AFAP-110 is overexpressed in prostate carcinoma, contributes to tumorigenic growth, and regulates focal contacts in prostate cancer cells.

\section{Results}

Expression of AFAP-110 in normal and pathologic prostate tissues. To investigate expression of AFAP-110 in normal prostate glands, benign prostatic hyperplasia $(\mathrm{BPH})$, and different stages of prostate cancer, we performed immunohistochemical staining on tissue arrays containing specimen cores of normal prostate $(n=40), \mathrm{BPH}(n=62)$, and prostate carcinoma $(n=198)$ with different Gleason scores, which positively correlate with the aggressiveness of the disease. Representative images of the immunohistochemical staining of normal and tumor tissues are shown in Figure 1. Quantification of staining intensity and its correlation with the pathological characteristics, as described in Methods, is summarized in Tables 1 and 2. Prostate smooth muscle tissues expressed high levels of AFAP-110 (Figure 1A), a result consistent with previous findings in muscle cells (6), and thus these tissues served as a positive internal control. No immunoreactivity was observed in stromal connective tissues, and thus these tissues served as internal negative controls. In normal prostatic glands (Figure 1B), no staining or occasional weak staining of AFAP-110 was observed in either the luminal secretory cell layer or the basal layer. In BPH epithelium (Figure 1C), the nonproliferating secretory cells were not immunoreactive; however, most cells in the basal layers were weakly to moderately stained. Recent studies suggest that aberrant proliferation and differentiation of basal layer cells contribute to both BPH and prostate cancer (22-26). Therefore, the expression of AFAP-110 in the basal epithelial cells of BPH suggests that AFAP-110 may play a role in the pathologic proliferation and/or differentiation of the basal cells in the prostatic glands.

In contrast to normal prostatic epithelia and $\mathrm{BPH}$, the majority of prostate cancer tissues exhibited a moderate to strong intensity of staining, while surrounding connective tissues had no observable immunoreactivity. In addition, the expression of AFAP-110 was higher in more aggressive tumors with higher Gleason scores $(\geq 7$; Figure $1 \mathrm{E})$ than in less aggressive tumors with lower Gleason scores ( $<7$; Figure 1D). Table 1 summarizes the differential AFAP-110 expression in normal prostate, $\mathrm{BPH}$, and carcinoma tissues. The immunostaining intensity of AFAP-110, as indicated by immunostaining scores, was significantly elevated in prostate cancer specimens compared with the normal prostate epithelium $(P<0.001)$. Furthermore, increasing AFAP-110 expression directly correlated with the increasing Gleason scores of the tumors $(P=0.015$; Table 2$)$. These results demonstrate that AFAP- 110 expression is upregulated 


\section{Table 1}

Staining intensity of AFAP-110 in normal and neoplastic prostate tissues

\begin{tabular}{|c|c|c|c|c|c|c|}
\hline \multirow{2}{*}{$\begin{array}{l}\text { Pathological } \\
\text { characterization }\end{array}$} & \multicolumn{4}{|c|}{ No. in intensity category/total number (\%) } & \multirow[t]{2}{*}{$\begin{array}{c}\text { ISS } \\
\text { (mean }[95 \% \mathrm{CI}] \text { ) }\end{array}$} & \multirow[t]{2}{*}{$\begin{array}{l}P \text { versus } \\
\text { normal }\end{array}$} \\
\hline & Negative, ISS: $0-0.5$ & Weak, ISS: 0.5-1.0 & Moderate, ISS: 1.0-1.5 & Strong, ISS: 1.5-2.0 & & \\
\hline Normal & $25 / 40(63 \%)$ & $15 / 40(37 \%)$ & $0 / 40(0 \%)$ & $0 / 40(0 \%)$ & $0.45(0.37-0.53)$ & $\mathrm{N} / \mathrm{A}$ \\
\hline $\mathrm{BPH}$ & $25 / 62(40 \%)$ & $35 / 62(56 \%)$ & $2 / 62(4 \%)$ & $0 / 62(0 \%)$ & $0.53(0.47-0.59)$ & 0.11 \\
\hline Cancer GS <7 & 1/67 (2\%) & 15/67 (22\%) & 38/67 (57\%) & 13/67 (19\%) & $1.20(1.13-1.27)$ & $<0.001$ \\
\hline Cancer GS $\geq 7$ & $0 / 131(0 \%)$ & $14 / 131(11 \%)$ & $73 / 131(56 \%)$ & $44 / 131(33 \%)$ & $1.38(1.28-1.48)$ & $<0.001$ \\
\hline
\end{tabular}

The immunostaining scores (means and 95\% Cls) for each pathological group and the numbers and percentages of tissues falling into each category of staining intensity are presented. GS, Gleason score; ISS, immunostaining score. $P$ values were determined using 2 -tailed Student's $t$ test.

in prostate cancer compared with nonmalignant tissues and increasing expression directly correlates with increasing Gleason score, which is a powerful predictor of disease progression.

AFAP-110 expression in prostate cell lines. To determine potential roles of increased AFAP-110 expression in prostate cancer, we first examined AFAP-110 levels in prostate cancer cell lines, DU145, LNCaP, and PC3, as well as an immortalized nontumorigenic human prostate epithelial cell line, RWPE-1 (27). Expression of AFAP-110 was extremely low in RWPE- 1 cells (Figure 2A). In contrast, AFAP-110 was greatly increased in all 3 human prostate cancer cell lines. This result is consistent with the expression of AFAP-110 observed in normal prostate epithelia and carcinoma tissues from tissue arrays.

Effects of AFAP-110 downregulation on cell proliferation, anchorageindependent growth, tumor incidence, and tumor growth in an orthotopic mouse model. To investigate the importance of AFAP-110 in prostrate cancer tumorigenesis and/or progression, we transfected PC3 cells with a plasmid directing the expression of either an siRNA specifically targeting human AFAP- 110 mRNA or a scrambled control siRNA and established stable sublines as described in Methods. Several stable clones with reduced AFAP-110 were obtained and further analyzed. Levels of AFAP-110 protein were reduced $80 \%$ in clone 309 and $70 \%$ in clone B11 compared with parental or scrambled control cells (Figure $2 \mathrm{~B}$ ). Both clones were stable in culture, maintaining a low AFAP-110 level for at least 22 passages in medium containing selection agents. Similar passages of these 2 clones were used in all the following studies.

To determine the effect of AFAP-110 downregulation on the tumorigenic properties of PC3 cells, we first assessed the in vitro proliferation and anchorage-independent growth of AFAP-110 siRNA clones. The clones with reduced AFAP-110 expression were significantly reduced in their proliferation rates compared with parental control cells; mean doubling time in hours (95\% confidence interval [CI]): parental, 19.4 (19.1-19.7); scrambled, 20.4 (19.8-21.0), $P=0.07$; clone 309, 26.9 (26.4-27.4), $P<0.001$; clone B11, 25.3 (24.9-25.7), $P<0.001$ (Figure 2D). The abilities of the cells to form colonies in soft agar are shown in Figure 3, A and B. Compared with parental cells, clone 309 and B11 formed significantly fewer colonies - mean number of colonies/ well (95\% CI): parental, 230 (212-248); scrambled, $225(215-235), P=0.7$; clone 309, 11 (4-18), $P<0.001$; clone B11, 20 (16-24), $P<0.001-$ indicating

\section{Table 2}

a decrease in anchorage-independent growth, a characteristic of tumorigenic cells. Colony formation was not increased in AFAP110-downregulated clones after 2 weeks of additional incubation, indicating that the change could not be attributed to the decreased proliferation rates of the siRNA-expressing clones.

We next investigated the effects of AFAP-110 downregulation on tumor incidence and growth in an orthotopic nude mouse model. Cells of different groups were implanted into the prostates of male nude mice as described in Methods. Four weeks after injection, prostates of the nude mice were excised, and tumor incidences and weights were determined. The results are summarized in Table 3. All (100\%) of the mice that were orthotopically injected with parental and scrambled control cells developed tumors in their prostates, with no statistically significant difference in tumor weight between these 2 groups. However, mice implanted with AFAP110-downregulated clones developed either no tumor (clone 309) or significantly smaller tumors relative to control groups (clone B11). These results suggest an important role of AFAP-110 in the tumorigenic growth of prostate cancer cells.

To verify the specificity of the siRNA in downregulating AFAP110 , we reintroduced a GFP-tagged chicken AFAP-110 into the siRNA clone 309 . The chicken AFAP-110 is highly homologous to its human ortholog (6) but does not contain the sequence to which the siRNA was designed. Stable subclones of clone 309 that ectopically express GFP-tagged wild-type chicken AFAP110 were established as described in Methods. Two representative clones, 309-AFAPGFP6 and 309-AFAPGFP7, are shown in Figure $2 \mathrm{C}$. We next compared their growth properties with those of parental cells. Ectopic expression of GFP-AFAP-110 rescued cell proliferation rates to a level comparable to that of parental cells - mean doubling time in hours (95\% CI): clone 309-AFAPGFP6, 20.6 (20.0-21.2), $P=0.1$; clone 309-AFAPGFP7, 20.7 (19.7-21.7) (Figure 2D) - and the ability of the cells to form colonies in soft

Association of the expression of AFAP-110 with prostate cancer progression
Pathological characterization

Cancer GS $<7$

Cancer GS $\geq 7$
No. of staining intensity/total number (\%) Low-expression, ISS $<1.0$

$16 / 67(24 \%)$

$14 / 131(11 \%)$
High-expression, ISS $\geq 1.0$ $51 / 67(76 \%)$ $117 / 131(89 \%)$
The staining intensity of tissue samples was classified to low-expression group (ISS <1.0) or highexpression group (ISS $\geq 1.0$ ) as described in Methods. The number and percentage of samples falling into each subgroup are presented. $P=0.015$, Pearson $\chi^{2}$ test. 
A

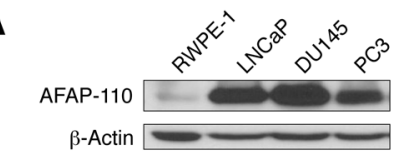

B

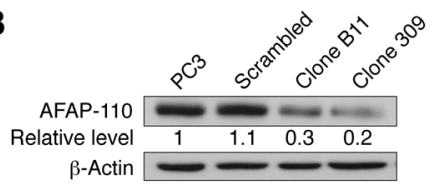

C

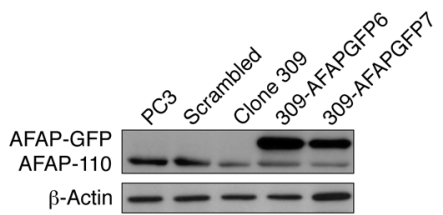

\section{Figure 2}

Downregulation of AFAP-110 in a prostate cancer cell line. (A) AFAP-110 protein levels in cancer cell lines LNCaP, DU145, and PC3 and a normal human prostate epithelial cell line, RWPE-1. Western blotting was performed with a monoclonal mouse anti-AFAP-110 antibody. (B) AFAP-110 expression in siRNA-expressing clones. PC3 cells were transfected with a plasmid containing an siRNA sequence targeting human AFAP-110 or a scrambled control sequence. Stable clones were established as described in Methods. Western blotting was performed with cell lysates from PC3 cells, 1 scrambled subclone, and 2 stable clones with siRNA (i.e., 309 and B11). The membranes were stripped and blotted with a monoclonal mouse anti- $\beta$-actin antibody. $\beta$-Actin expression was used as a loading control. Results from 1 of 2 independent experiments are shown. (C) Ectopic expression of GFP-tagged chicken AFAP-110. AFAP-110-downregulated clone 309 was transfected with a plasmid harboring a gene encoding a GFP-tagged chicken AFAP-110, and stable clones (309-AFAPGFP6 and 309-AFAPGFP7) were established. Western blotting was performed. $\beta$-Actin expression was used as a loading control. Results from 1 of 2 independent experiments are shown. (D) In vitro proliferation of cells as measured by counting using a hematocytometer. Parental PC3 cells (diamonds), scrambled siRNA control cells (squares), clone 309 (triangles), clone B11 (Xs), clone 309-AFAPGFP6 (asterisks), and clone 309-AFAPGFP7 (circles). Values are expressed as mean and $95 \% \mathrm{Cl}$ of experiments performed in triplicate.

agar was restored to a level comparable to that of the parental cells; mean number of colonies (95\% CI): clone 309-AFAPGFP6, 208 $(197-219), P=0.1 ; 309$-AFAPGFP7, 194 (168-220), $P=0.1$ (Figure 3 , $A$ and $B)$. These data demonstrated that results of AFAP-110 downregulation were not due to a nonspecific siRNA effect.

Suppression in cell adhesion to ECM components and migration mediated by AFAP-110 downregulation. Interactions between cancer cells and extracellular substrates are essential for cell growth, migration, and survival in the process of tumor development and spreading $(28,29)$. In tissue culture, we noted that AFAP-110downregulated cells were much more readily detached relative to parental and scrambled control cells. While PC 3 cells assumed an elongated morphology, AFAP-110 clones exhibited a roundedup, balloon-like shape. These features suggested that decreased AFAP-110 expression may cause an interruption of cell-ECM adhesions, contributing to the reduction in proliferation in vitro and tumorigenic growth in vivo.

To examine this possibility, adhesion assays were performed to 2 important components of the basement membrane, laminin and type IV collagen. The results are shown in Figure 4. Downregulation of AFAP-110 in PC3 cells induced a decrease in cell adhesion to both laminin and type IV collagen: mean percentage of cells attached to laminin ( $95 \% \mathrm{CI})$ : parental, $46 \%(35 \%-66 \%)$; scrambled, $42 \%(24 \%-59 \%), P=0.7$; clone $309,5 \%$ (1\%-8\%), $P=0.01$; clone B11, $4 \%(1 \%-7 \%), P=0.01$ (Figure $4 \mathrm{~A}$ ); mean percentage of cells attached to type IV collagen ( $95 \% \mathrm{CI})$ : parental, $95 \%(88 \%-102 \%)$; scrambled,
99\% (87\%-110\%), $P=0.6$; clone 309, $55 \%(49 \%-60 \%), P=0.01$; clone B11, $63 \%(53 \%-73 \%), P=0.007$ (Figure 4B). In addition, migration of cells with reduced AFAP-110 expression was significantly decreased, as assessed after 24 hours by modified Boyden chamber assays: mean number of migrated cells/ field (95\% CI): parental, 192 (184-200); scrambled, 149 (129-169), $P=0.06$; clone 309, 1 (0.3-1.7), $P<0.001$; clone B11, 3 (1.7-4.3), $P<0.001$ (Figure 4, C and $\mathrm{D})$. The reduction in cell migration was not reflective of decreased proliferation rates, as the difference in cell numbers at 24 hours in proliferation assay between AFAP-110-downregulated clones and parental cells was not statistically significant (clone 309 , $P=0.08$; clone $\mathrm{B} 11, P=0.9$; Figure $2 \mathrm{D}$ ).

Effects of AFAP-110 downregulation on focal adhesion and integrin $\beta 1$ expression. The formation of focal adhesion complexes is a prerequisite for cell-ECM adhesion and migration $(30,31)$. The decrease in cell adhesion and migration capabilities of clones with AFAP-110 downregulation may result from alterations in focal adhesion functions. We thus used immunofluorescence staining to examine the effects of AFAP- 110 downregulation on focal adhesions, as well as on the actin filament organization. As shown in Figure 5A, PC3 cells and scrambled control cells spread out on the slide chambers in which they were grown and assumed fibroblast-like shapes with stress fibers and cortical actin filaments, which were stained by fluorescence-labeled phalloidin. In parental cells, multiple focal adhesion structures were formed at plasma membranes, as visualized by staining for vinculin, an abundant cytoskeleton protein localized at focal adhesions. In marked contrast, clones with decreased AFAP-110 had a rounded-up morphology with disturbed actin filament organization and failed to form focal adhesions.

Downregulation of AFAP-110 does not affect expression or activity of tyrosine kinases associated with focal adhesions. Several molecular mechanisms might contribute to the defect in focal adhesions in AFAP-110-downregulated clones. We first investigated the effect of AFAP-110 on the expression and phosphorylation status of focal adhesion kinase (FAK), a tyrosine kinase that is essential in focal adhesion turnover and is phosphorylated by Src at multiple sites, including tyrosine 861 (Y861). We utilized PC3 cells that express a constitutively active form of chicken Src ( $\mathrm{Src}$ Y527F) as a positive control. As shown in Figure $5 \mathrm{~B}$, no changes were observed in total or phospho-FAK (Y861). Next, expression of total and activated Src was examined, again with no differences observed. Consistent with these results, no change in phosphorylation of the Src substrate paxillin was observed. These results demonstrated that AFAP-110 downregulation did not affect Src or FAK activity, nor the expression of several focal adhesion-associated structural proteins, indicating that decreased focal adhesions resulted from alterations in other focal adhesion components. 
A
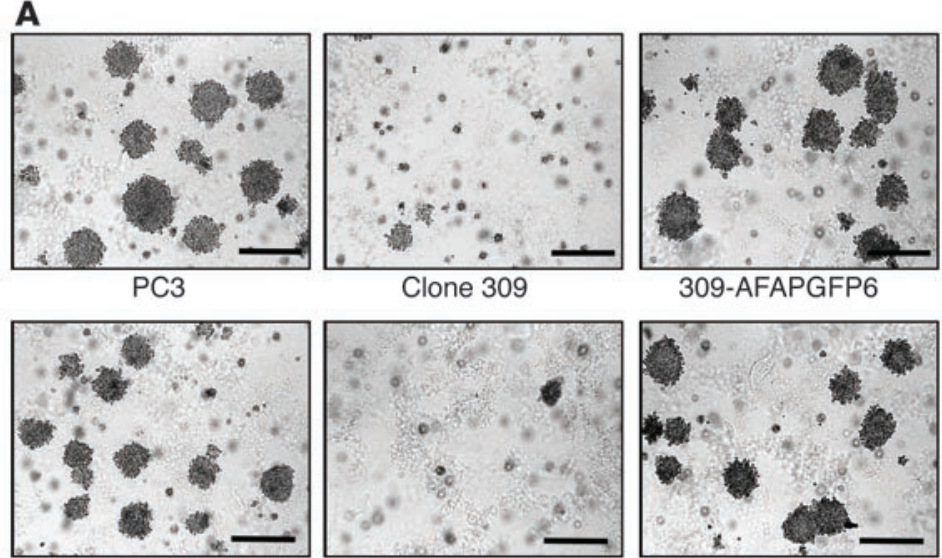

Scrambled

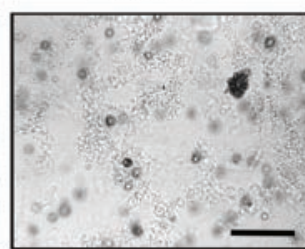

Clone B11
309-AFAPGFP6

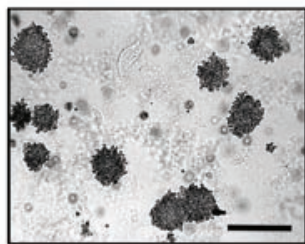

309-AFAPGFP7

B

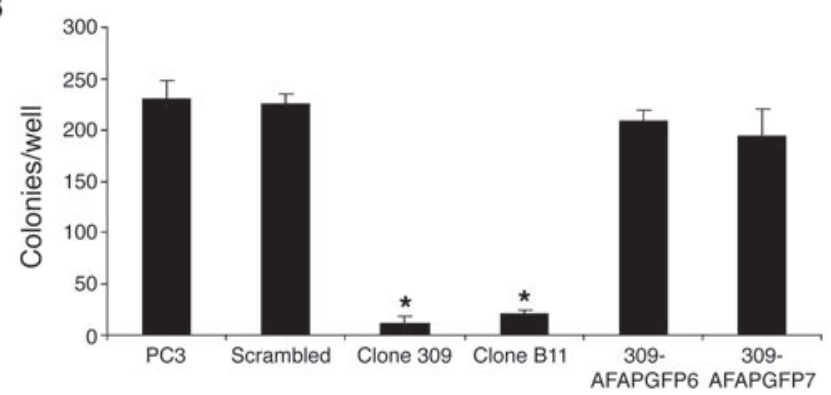

\section{Figure 3}

Effects of AFAP-110 downregulation on the ability to form colonies on soft agar. The colony-forming assay was performed by seeding equal amount of cells of different groups in soft agar in 6-well tissue culture plates in triplicate. Numbers of colonies with a diameter greater than $20 \mu \mathrm{m}$ were quantified after 2 weeks. (A) Representative images of colonies in soft agar. Scale bars: $50 \mu \mathrm{m}$. (B) Numbers of colonies per well are expressed as means and $95 \% \mathrm{Cls}$ of experiments performed in triplicate ( 2 independent experiments were performed); bars correspond to $95 \%$ Cl. ${ }^{\star} P<0.001,2$-tailed Student's $t$ test.
Downregulation of AFAP-110 decreases expression of integrins. We next examined expression of integrins that are essential for the establishment of focal contacts. Specifically, we examined the expression of integrin $\beta 1$, the most abundant integrin subunit found in PC3 cells (32), by immunoblotting and immunofluorescence staining (Figure 5, C and D). Compared with parental and scrambled controls, AFAP-110-downregulated clones were substantially reduced in the expression of integrin $\beta 1$, while the expression of cell-cell adhesion molecule E-cadherin was not affected. Thus, the defect in focal adhesion formation in AFAP-110-downregulated clones correlates with the decreased level of integrin $\beta 1$.

Effect of ectopic expression of wild-type and truncation mutants of AFAP-110 in focal adhesion and integrin $\beta 1$ expression. To determine which functional domain of AFAP-110 is required for its ability to regulate focal adhesion and/or integrin $\beta 1$, we reintroduced GFP-tagged wild-type chicken AFAP-110 as well as its mutant variants, illustrated in Figure 6A, into the siRNA clone 309. Stable subclones of clone 309 that ectopically express GFP-tagged wild-type or mutant chicken AFAP-110 were established as described in Methods. As shown in Figure 6B (top panel), expression of both wild-type and mutant constructs was achieved while maintaining reduced levels of endogenous AFAP-110. We next examined the formation of focal adhesions and expression of integ$\operatorname{rin} \beta 1$ in these subclones. Reintroduction of wildtype AFAP-110 into clone 309 restored the protein levels of integrin $\beta 1$ in siRNA clone 309 (Figure 6B) and induced a morphology similar to that of parental cells (Figure 6C). Expression of the mutation variant defective in the ability of AFAP-110 to interact with Src (309-AFAP71A) also restored inte- grin $\beta 1$ expression (Figure 6B) as well as parental cell shape and focal adhesions (Figure 6C). These results further indicate that the effects of AFAP-110 in prostate cancer might be Src independent. However, cells with ectopic expression of mutant variant AFAP-110 lacking PH1 domain, and thus the ability of AFAP-110 to associate with $\mathrm{PKC}$, failed to restore integrin $\beta 1$ expression, focal adhesions, and cell morphology (Figure 6, B and C). These data suggest that the role of AFAP-110 in regulating focal contacts is mediated by a PKC-dependent mechanism.

\section{Discussion}

This study provides the first evidence to our knowledge that AFAP110 plays an important role in the development and progression of a human cancer, prostate adenocarcinoma. We observed, by prostate tissue array analyses, that AFAP-110 was significantly upregulated in prostate carcinoma, whereas in normal prostate
Table 3

Tumor incidence and growth following intraprostate implantation of tumor cells into nude mice

\begin{tabular}{lccccc} 
Cell line & Incidence & \multicolumn{3}{c}{ Tumor mass $(\mathbf{m g})$} & Pversus PC3 \\
& & Mean & Median & Range & \\
PC3 & $5 / 5$ & 440 & 420 & $270-650$ & N/A \\
Scrambled & $5 / 5$ & 516 & 540 & $120-820$ & 0.6 \\
Clone 309 & $0 / 5$ & N/A & N/A & N/A & N/A \\
Clone B11 & $4 / 5$ & 81 & 85 & $45-110$ & 0.007
\end{tabular}

Prostates were excised 4 weeks after mice were injected with cells of different groups ( $n=5$ per group). N/A, not applicable. Tumor incidence and the weights of tumor mass of each group are presented. The differences in tumor weights were determined using Mann-Whitney $U$ test. 
A

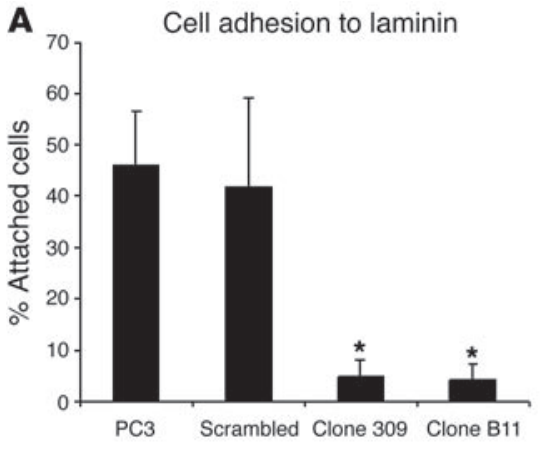

C

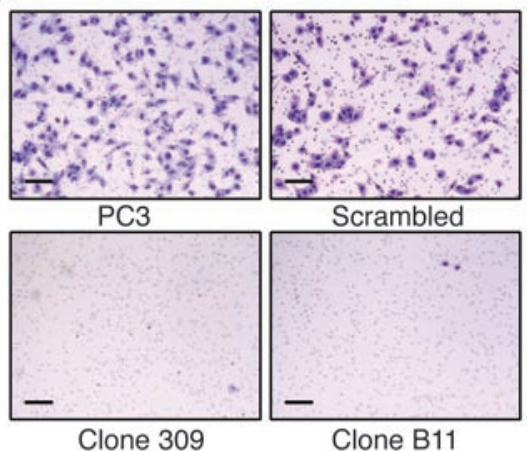

B Cell adhesion to type IV collagen

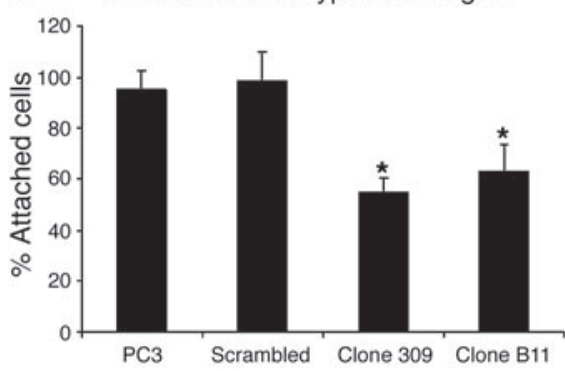

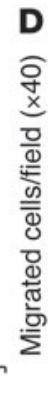

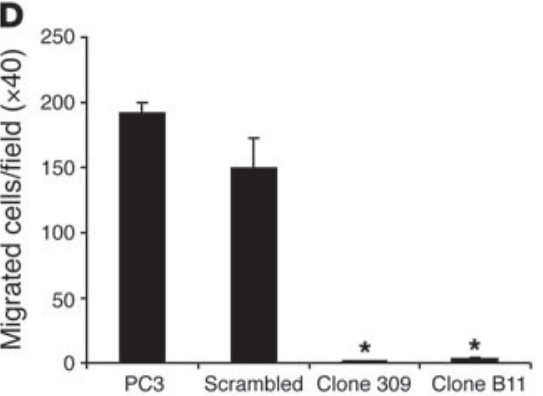

\section{Figure 4}

Effects of AFAP-110 downregulation on cell adhesion to ECM components and cell migration. (A and B) AFAP-110 downregulation-induced inhibition of cell adhesion to extracellular components. The adhesion assay was performed by seeding equal amount of cells of different groups in 96-well tissue culture plates coated with laminin (A) or type IV collagen (B). Cells were washed 3 times with PBS at 1 hour after plating, and the percentage of attached cells to total cell inputs was determined using methylthiazole tetrazolium (MTT) staining. Means and $95 \% \mathrm{Cls}$ of the percentages of 3 independent experiments are presented; bars correspond to $95 \%$ Cl. ${ }^{*} P<0.05$, 2-tailed Student's $t$ test. (C and D) AFAP-110 downregulation-induced inhibition of cell migration. Migration assays were performed using Boyden chambers. Cells that migrated through 8-um pores to the bottom side of the inserts were stained and quantified. (C) Representative images of migrated cells of different groups. Scale bars: $100 \mu \mathrm{m}$. (D) Numbers of migrated cells per field under $\times 40$ magnificent microscope were quantified and expressed as means and $95 \%$ Cls of 3 independent experiments; bars correspond to $95 \%$ Cl. ${ }^{*} P<0.001,2$-tailed Student's $t$ test.

epithelia, the expression was virtually undetectable. In addition, AFAP-110 expression positively correlated with the aggressiveness of prostate cancer. In agreement with the expression pattern in normal and malignant prostate tissues, AFAP-110 expression was low in nontumorigenic prostate epithelial cells and substantially higher in several prostate cancer cell lines. Downregulation of AFAP-110 in prostate cancer cells led to an inhibition of cell proliferation, anchorage-independent growth, and tumor incidence and growth in an orthotopic nude mouse model. These findings strongly indicate that AFAP- 110 overexpression contributes to the tumorigenic growth of prostate cancer. As of yet, the mechanism of AFAP- 110 overexpression in prostate cancer is unclear. There is no evidence of a genetic lesion, such as gene amplification, of chromosome 4p16.1, the location of human AFAP-110. The expression of AFAP-110 protein is likely to be regulated at transcription and mRNA levels, and further investigation is required to elucidate mechanisms underlying the upregulation of AFAP-110 in prostate cancer. The expression pattern and function of AFAP-110 could be tissue specific, as most colon tumor cells we examined express extremely low levels of AFAP-110 (J. Zhang and G.E. Gallick, unpublished observations). However, an upregulation of AFAP-
110 in some stage I invasive ductal breast carcinomas was observed (33), suggesting that aberrant expression of AFAP-110 may play a role in other types of cancers.

Previous work demonstrated that AFAP-110 has an intrinsic ability to induce the formation of lamellipodia and podosomes, both of which are actin-rich structures that are localized at cell membrane and are important in cell motility and invasiveness $(3,16)$. Our data show a direct role of AFAP-110 in regulating cell-matrix adhesions and migration in prostate cancer cells. Downregulation of AFAP110 significantly decreased prostate cancer cell adhesion to ECM components, as well as significantly inhibiting cell migration. While focal adhesions were frequently observed in PC3 prostate cancer cells, AFAP-110-knockdown clones failed to form focal adhesions, and ectopic expression of avian AFAP-110 restored these structures. These results strongly indicate that AFAP-110 regulates focal adhesions, which are essential for cell-matrix adhesion, as well as a variety of other cell activities.

Several molecular mechanisms might contribute to the defect in focal adhesions in AFAP-110-downregulated clones. One possibility is that AFAP-110 is critical in regulating stress fibers involved in formation of focal adhesions. However, AFAP-110 regulation of actin cytoskeleton integrity may also occur through an indirect mechanism. The intrinsic ability of AFAP-110 to induce a malignant transformation-like morphology is dependent on the integrity of both SH3binding motif and the amino-terminal $\mathrm{PH}$ domain (3), which direct the association to Src and PKC, respectively. Therefore, siRNAmediated inhibition of AFAP-110 expression may have altered the functions of these signaling molecules that are known to be important in regulating focal adhesions.

In agreement with this possibility, our data demonstrated that the amino-terminal PH1 domain of AFAP-110 is required for its function in regulating cell morphology and focal adhesion formation, suggesting that downregulation of AFAP-110 may have altered PKC-regulated signal transduction pathways. The PH1 domain of AFAP-110 has been demonstrated to direct the association of this protein with several PKC family members, including classic PKCs $(\alpha, \beta, \gamma)$ and atypical PKC (PKC $\lambda$ ), among which only 2 are found to be expressed in PC3 cells: PKC $\alpha$ and PKC $\lambda$ (34). PKC $\alpha$ is significantly overexpressed in prostate carcinoma compared with nonmalignant lesion and plays important roles in the malignant progression of the disease $(17,19)$, and preliminary work in our laboratory suggests that, indeed, altered expression of AFAP-110 affects signal transduction pathways mediated by PKC $\alpha$, including signals that regulate the expression of ECM components.

Further, AFAP-110 has an intrinsic ability to activate Src tyrosine kinase. Alteration in AFAP-110 configuration, such as deletion of the leucine zipper domain or phosphorylation by $\mathrm{PKC}$, induces the activation of Src. Earlier studies indicate that AFAP-110 modulates 

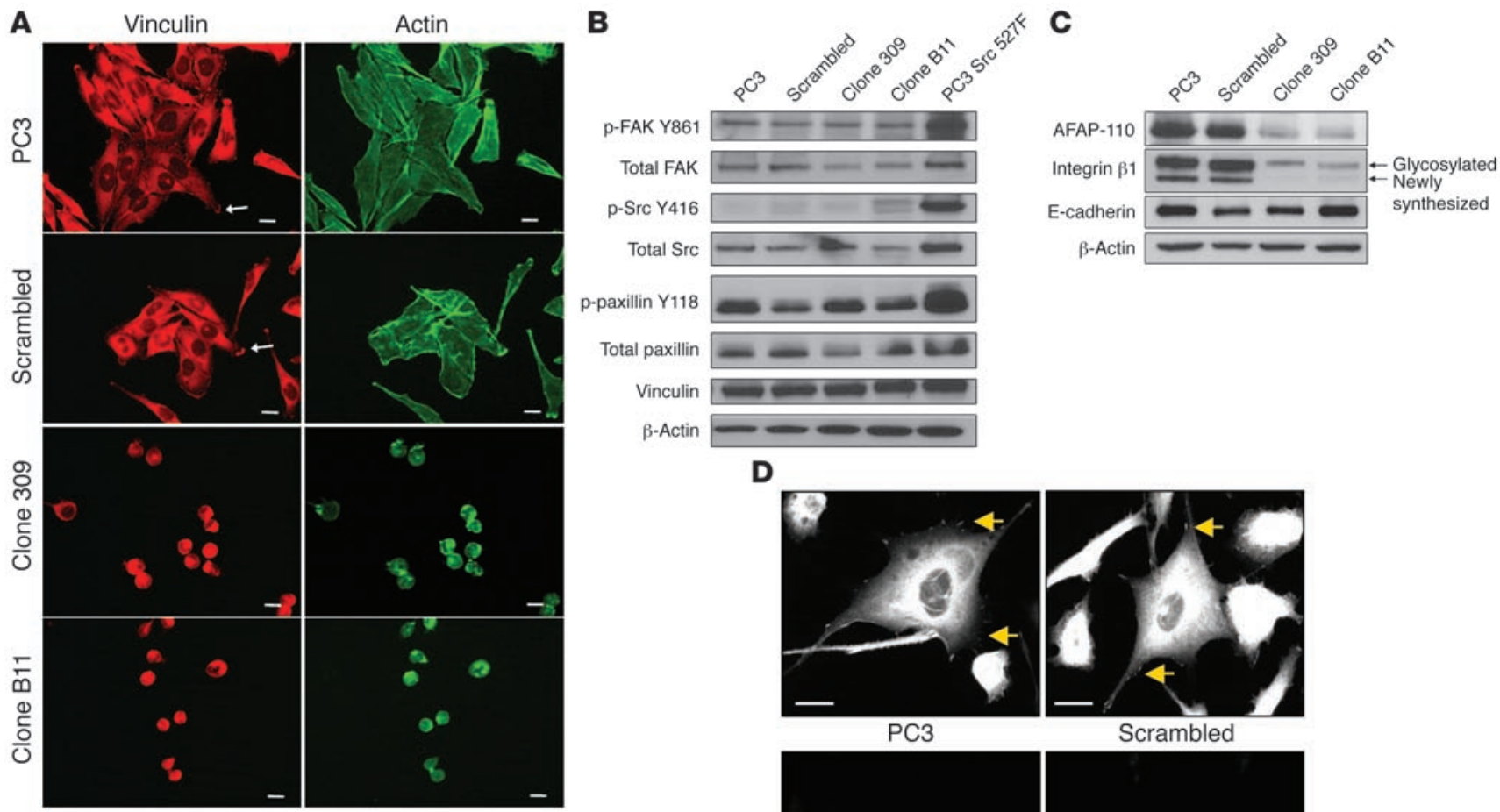

D

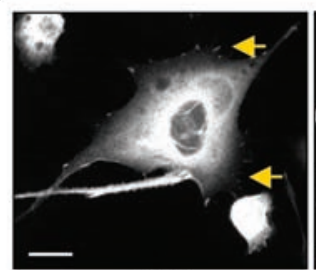

PC3

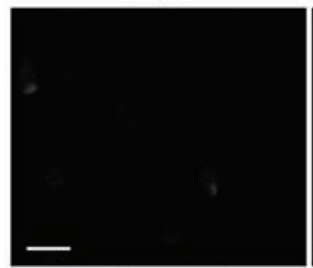

Clone 309

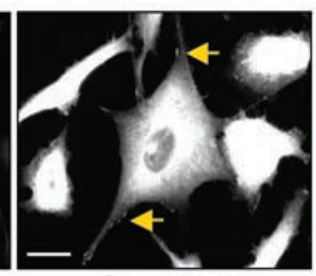

Scrambled

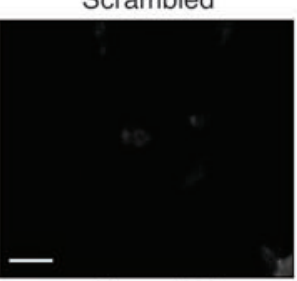

Clone B11

\section{Figure 5}

Effects of AFAP-110 downregulation on focal adhesion and integrin $\beta 1$ expression. (A) Loss of focal adhesion structures (white arrows) mediated by AFAP-110 downregulation. Immunofluorescence staining of vinculin, a cytoskeletal protein localized at focal adhesion structures, was performed using a monoclonal anti-vinculin primary antibody and a goat anti-mouse Alexa Fluor 594-conjugated secondary antibody (red). Actin was stained by Alexa Fluor 488-conjugated phalloidin (green). Representative images of 2 independent experiments are shown; scale bars: $20 \mu \mathrm{m}$. (B) Expression and activity of Src in phosphorylating adhesion-associated substrates. Western blotting of cell lysates was performed with monoclonal mouse antibodies selective for phospho-FAK tyrosine 861 (Y861), phospho-c-Src tyrosine 418 (Y418), phospho-paxillin tyrosine 118 (Y118), as well as antibodies recognizing total protein Src, FAK, paxillin, and vinculin. Cell lysate from PC3 cells that express a constitutively active form of Src (PC3 Src 527F) was used as a positive control. Membranes were probed for $\beta$-actin as loading control. Results from 1 of 2 independent experiments are shown. (C) Immunoblotting with antibodies against AFAP-110, integrin $\beta 1$, and E-cadherin. $\beta$-Actin expression was used as a loading control. (D) Immunofluorescence staining of integrin $\beta 1$ was performed as described in Methods, using a monoclonal anti-integrin $\beta 1$ primary antibody and a goat anti-mouse Alexa Fluor 594-conjugated secondary antibody (red). Images were converted to grayscale pictures for best visualization of the localization of integrin $\beta 1$ at focal adhesion structures in PC3 and scrambled control cells (yellow arrows). Representative images of 2 independent experiments are shown; scale bars: $20 \mu \mathrm{m}$.

the formation of lamellipodia and podosomes via activating $\operatorname{Src}(3)$. However, in the present study, we did not observe notable changes in Src activity or its ability to phosphorylate downstream signaling molecules known to play important roles in focal adhesion construction, such as FAK and paxillin $(35,36)$. Furthermore, transfection of a mutant variant of chicken AFAP-110 that is defective in binding to Src successfully restored focal adhesions and integrin $\beta 1$ expression, demonstrating the AFAP-110-mediated regulation of focal adhesion in PC 3 cells is not affected by its ability to associate with and to activate Src. These data suggest that AFAP-110 may function independently of Src in PC3 prostate cancer cells.

The finding of the ability of AFAP-110 to regulate the expression of integrin $\beta 1$ in prostate cancer cells is novel and provides a potential molecular basis for the direct involvement of AFAP110 in the focal adhesion signaling machinery. Precisely how integrin $\beta 1$ is regulated by AFAP-110 remains to be studied. We found comparable mRNA levels of integrin $\beta 1$ in PC3 parental cells and AFAP-110-downregulated clones (data not shown), suggesting that AFAP-110 participates in posttranscriptional regulation of this protein. Interestingly, other investigators have demonstrated the regulation of integrin $\beta 1$ by PKC $\alpha$ (37). PKC $\alpha$ directly associates with integrin $\beta 1$, and the assembly of the PKC $\alpha$-integrin $\beta 1$ complex is correlated with recruitment and phosphorylation of the actin-binding protein ezrin, which may direct intracellular membrane association (38). It is possible that AFAP-110, by directly interacting with PKC $\alpha$, actin cytoskeleton, and potentially phospholipids, can facilitate the localization and transportation of the PKC $\alpha$-integrin $\beta 1$ complex to cellular and subcellular membranes such as lysosomes, affecting the stability of integrin $\beta 1$ proteins. 


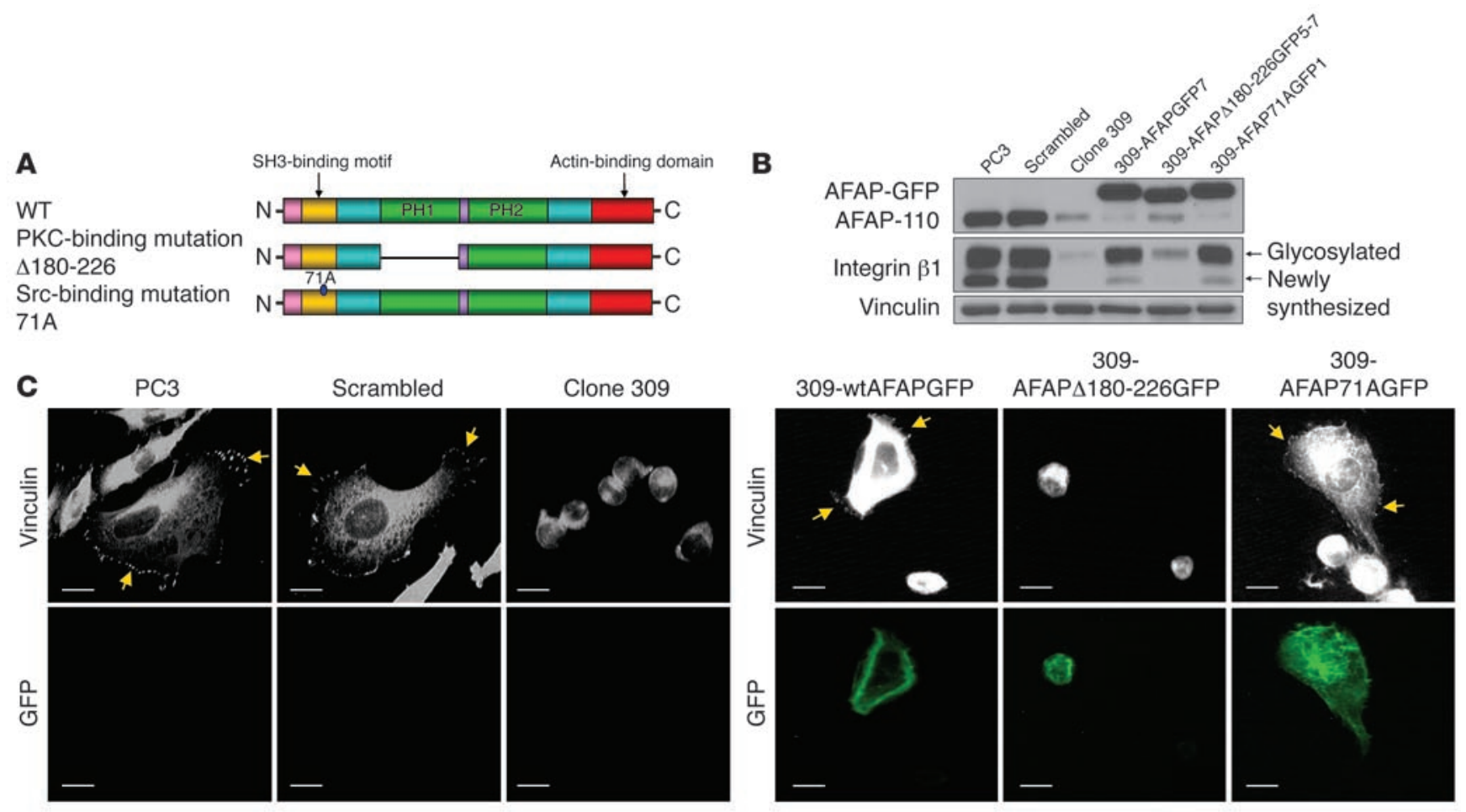

Figure 6

Restoration of integrin $\beta 1$ expression and focal adhesions by ectopic expression of wild-type AFAP-110 and functional mutant variants. (A) Schematic representation of wild-type AFAP-110 and functional mutant variants. Deletion of the PH1 domain (AFAP-110 $\Delta 180-226)$ abolishes the association of AFAP-110 with PKC. A single amino acid mutation that changed a proline residue to an alanine (AFAP71A) at the SH3-binding motif abrogates the ability of AFAP-110 to interact with Src. (B) Immunoblotting with antibodies against AFAP-110 and integrin $\beta 1$. Ectopic expression of GFP-tagged chicken wild-type AFAP-110 and mutant variants was visualized as bands that localized slightly higher than endogenous AFAP-110 on the membrane. Vinculin expression was used as a loading control. (C) Immunofluorescence staining of vinculin was performed using a monoclonal anti-vinculin primary antibody and a goat anti-mouse Alexa Fluor 594-conjugated secondary antibody (red). Images were converted to grayscale for best visualization of focal adhesion structures (yellow arrows). Ectopic expression of GFP-tagged chicken wild-type AFAP-110 and mutant variants in AFAP-110-downregulated clone 309 were visualized as proteins emitting green fluorescence under microscope (green). Representative images are shown; scale bars: $20 \mu \mathrm{m}$.

Collectively, our results demonstrated that AFAP-110, an actin cytoskeleton-associated adaptor protein, is overexpressed in prostate carcinoma and plays an important role in tumorigenic properties of prostate cancer cells. We hypothesize the following model. AFAP-110 directly associates with PKC and the actin cytoskeleton, regulating PKC-mediated integrin cell-surface and intracellular localization, which are essential for focal adhesion formation and subsequent cell activities (Figure 7A). In normal and benign hyperplastic epithelium, AFAP-110 is expressed at very low levels, and mainly in the basal layer cells, facilitating the formation of focal adhesions where these cells interact with basal membrane components. Prostate cancer cells also directly interact with substratum, although they retain certain luminal features, such as expression of androgen receptors and prostate-specific antigens. During the development of prostate cancer, the expression of AFAP-110 increases, contributing to the alteration in the expression and function of integrins, which mediate cancer cell-ECM interactions and propagate signaling pathways essential in tumorigenic growth and invasive progression (Figure 7B). Given that this actin-associated adaptor protein is selectively upregulated in prostate carcinoma tissue compared with normal epithelium, and downregulation of this protein in cancer cells inhibits tumorigenicity and other malignant properties, AFAP-110 could be a novel diagnostic and prognostic marker for prostate carcinoma and may be a potential target for novel therapeutic agents for the disease.

\section{Methods}

Prostate tissue arrays. Human prostate tissue arrays were purchased from Cybrdi and US Biomax Inc. The prostate adenocarcinoma array obtained from Cybrdi (catalog CC19-01-003) contains tumor tissues from 29 individuals, spotted in duplicate, plus 5 spots of BPH tissues. Each array spot was $1.5 \mathrm{~mm}$ in diameter and $5 \mu \mathrm{m}$ in thickness. The normal prostate tissue array from Cybrdi (catalog CC19-11-001) comprised normal prostate tissues from 6 individuals, spotted in triplicate, plus BPH tissues from 3 individuals and adenocarcinoma tissues from 3 individuals, both spotted in duplicate. Each array spot was $1 \mathrm{~mm}$ in diameter and $5 \mu \mathrm{m}$ in thickness. Two prostate carcinoma arrays from US Biomax Inc. (catalog PR801, PR802) each contained 80 cores, each tissue spot representing 1 individual specimen. Prostate tissue from US Biomax Inc. (catalog BC19017) included 10 individual prostate carcinoma specimens and 23 prostatic hyperplasia specimens. Each specimen was represented by 2 cores from different tissue spots. Each array spot was $1.5 \mathrm{~mm}$ in diameter and $5 \mu \mathrm{m}$ in thickness. The normal/hyperplasia tissue array from US Biomax Inc. (catalog BC19111) comprised 30 cores, 6 cases of normal prostate tissue in triplicate as well 
A

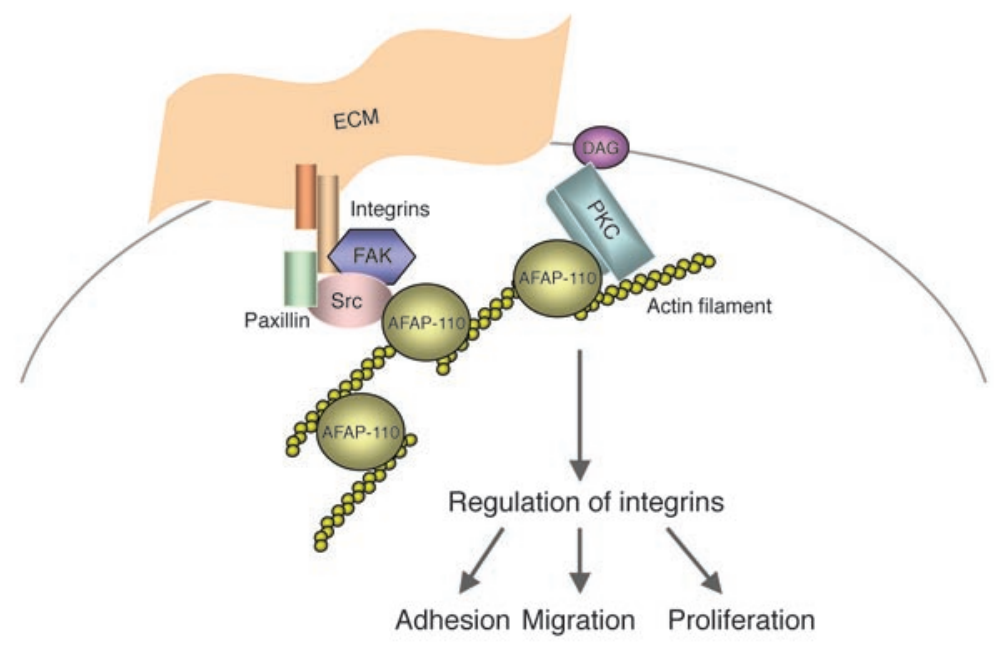

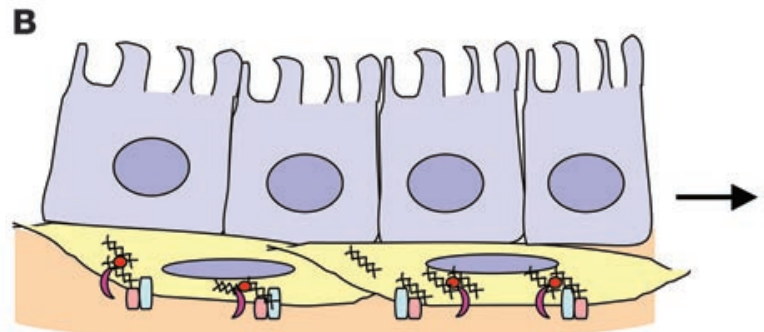

Normal epithelium

OAFAP-110

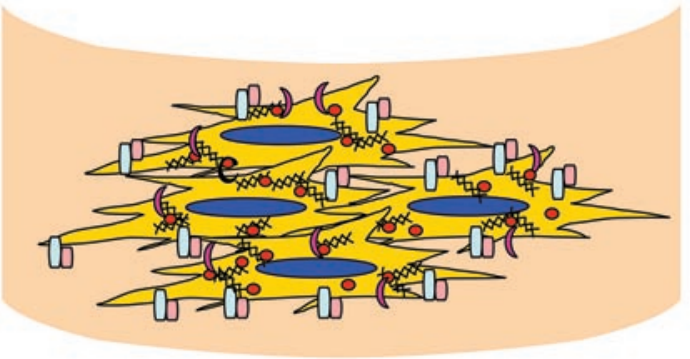

Prostate cancer

(PKC

Actin filament

\section{Figure 7}

Model for effects of AFAP-110 overexpression on deregulation of prostate cancer cell functions. (A) AFAP-110 directly associates with PKC and the actin cytoskeleton. Overexpression of AFAP-110 interferes with PKC signaling pathways that affect integrin expression and intracellular localization, which are essential for focal adhesion formation, and subsequent cell activities. (B) In normal and benign hyperplasic epithelium, AFAP-110 is expressed at a very low level, and mainly in the basal layer cells, facilitating the formation of focal adhesions where these cells interact with basal membrane components. During the development of prostate cancer, the expression of AFAP-110 increases, contributing to the alteration in the expression and function of integrins, which mediate cancer cell-ECM interactions and propagate signaling pathways essential in tumorigenic growth and invasive progression.

as 3 cases of prostate tumor and 3 cases of hyperplasia, both in duplicate. Each array spot was $1 \mathrm{~mm}$ in diameter and $5 \mu \mathrm{m}$ in thickness. The histological diagnosis and grading using the Gleason scoring system were supplied by the manufacturer. Detailed information for these arrays can be viewed at http://www.cybrdi.com/viewproduct.php?id=303 (normal/hyperplasia array); http://www.biomax.us/tissue-arrays/Prostate/BC19111 (normal/ hyperplasia array); http://www.biomax.us/tissue-arrays/Prostate/BC19017 (hyperplasia/carcinoma array); http://www.biomax.us/tissue-arrays/Prostate/PR801 (prostate carcinoma array); http://www.biomax.us/tissuearrays/Prostate/PR802 (prostate carcinoma array).

Immunohistochemistry. Immunohistochemical staining was performed as described previously (39). Briefly, paraffin-embedded tissue microarray sections were heated at $60^{\circ} \mathrm{C}$ for 1 hour before deparaffinization in xylene, followed by treatment with a graded series of alcohols $(100 \%, 95 \%$, and $80 \%$ ethanol [ $\mathrm{vol} / \mathrm{vol}]$ in double-distilled $\mathrm{H}_{2} \mathrm{O}$ ) and rehydration in PBS ( $\mathrm{pH} 7.5$ ). For antigen retrieval, the sections were submerged in a Borg Decloaker (Biocare Medical) in a pressure cooker for a total of 40 minutes (4 minutes actual cook time). After washing in PBS, endogenous peroxidases were blocked with $3 \%$ hydroxyl peroxide $\left(\mathrm{H}_{2} \mathrm{O}_{2}\right)$ in PBS for 12 minutes, followed by 3 washes in PBS. The sections were blocked with $4 \%$ fish gel in a humidified chamber for 20 minutes and then incubated with the primary antibody, anti-AFAP-110 (BD Biosciences), overnight at $4^{\circ} \mathrm{C}$. After washing with $\mathrm{PBS}$, the slides were incubated with biotinylated goat anti-mouse IgG (Biocare Medical) for 30 minutes at room temperature, followed by streptavidin-HRP (Dako) incubation for 30 minutes at room temperature. The chromogenic reaction was visualized by incubating the slides in with stable 3,3'-diaminobenzidine (DAB) solution (Invitrogen) for 10 minutes or until good color formation was observed by monitoring the reaction under a microscope. Positive staining appears brown. The sections were rinsed with distilled water, counterstained with Gill's hematoxylin solution for 1 minute, and mounted with Universal Mount (Research Genetics). Negative control samples were exposed to secondary antibody alone and demonstrated no specific staining.

Evaluation of immunostaining. The images of each tissue core on the microarrays, with exclusion of samples lost after the immunohistochemical staining procedure (final number of samples: normal prostate tissues, 40 ; prostatic smooth muscles, 3; BPH, 62; carcinoma, 198), were captured by a video camera (Sony DXC-990) mounted to a microscope (Nikon Microphot-FX) at $\times 100$ magnification. All pictures were taken at the same settings. The optical density of each image was obtained using the 
computer-based image analysis program Optimas 6.51 (Media Cybernetics). The immunostaining intensity of each sample was quantified as the average optical density readings of 10 randomly selected malignant tumor areas in prostatic carcinoma samples (or epithelium areas in normal and BPH tissues; or muscle cells in prostatic smooth muscle tissues). The immunostaining score for each specimen was calculated as the average immunostaining intensity of tumor area (or epithelium areas in normal and BPH tissues; or muscle cells in prostatic smooth muscle tissues) minus the average immunostaining intensity readings of stromal connective tissue area on the same specimen, which served as a blank control. Thus, the relative staining intensities of all tissue cores were normalized to account for background staining for further comparison. The immunostaining scores, ranging from 0 to 2.1 , were categorized as negative (0-0.5), weak (0.5-1), moderate (1-1.5), or strong (>1.5).

Cell culture. Human prostate cancer cell lines, PC3, LNCaP, and DU145, were maintained as monolayer cultures in DMEM F-12 (Life Technologies Inc.; Invitrogen) supplemented with 10\% FBS (HyClone). Human prostate epithelial cell line RWPE-1 cells were maintained in keratinocyte serum-free medium (Gibco; Invitrogen) supplemented with $50 \mu \mathrm{g} / \mathrm{ml}$ bovine pituitary extract, $5 \% \mathrm{~L}$-glutamine, and $5 \mathrm{ng} / \mathrm{ml} \mathrm{EGF}$. Cell cultures were incubated in $5 \% \mathrm{CO}_{2} / 95 \%$ air tissue culture incubators at $37^{\circ} \mathrm{C}$.

siRNA and plasmid constructs. siRNA expression plasmids were created as described previously (40) using the Ambion pSilencer 4.1 according to the manufacturer's directions. AFAP-110-specific target sequences were designed using the Ambion siRNA web design tool. The target sequence utilized was (3,052-3,072 bp) 5'-AAGCTGCCATTCTTCTTAACC-3'. A scrambled control sequence was created for this target as $5^{\prime}$-CAGTTCGATACTACTTCTCAC- $3^{\prime}$. Oligonucleotides corresponding to these sequences with flanking BamHI (nt 516) and HindIII (nt 463) ends were purchased from Invitrogen and ligated into the expression plasmid at compatible sites. Constructs were confirmed by DNA sequencing.

Transfection and creation of stable cell lines. Subconfluent PC 3 cells in 100-mm tissue culture dishes were transfected with $5 \mu \mathrm{g}$ of pSilencer4.1 plasmid, containing a hygromycin-resistant gene with either siRNA targeting AFAP-110 or a scrambled control sequence using FuGENE 6 Transfection Agent (Roche). Cells were then grown in DMEM-F12 medium containing $500 \mu \mathrm{g} / \mathrm{ml}$ hygromycin B (BD Biosciences - Clontech) for selection of stable transfectants. Single colonies were isolated and expanded for analyses. AFAP-110 expression in siRNA clones was determined by Western blot analysis as we described previously (41). Selected clones were maintained in DMEM-F12 medium containing $500 \mu \mathrm{g} / \mathrm{ml}$ hygromycin B.

The pEGFP-wtAFAP-110, pEGFP-AFAP71A, pEGFP-AFAP $\triangle 180-226$ plasmids were constructed and sequenced by Guappone et al. (8). These plasmids harbor a neomycin-resistant gene and a gene encoding GFPtagged wild-type or mutant chicken AFAP-110. The plasmids were transfected into one of the stable siRNA clones to restore AFAP-110 levels to at least those observed in parental cells. Stable transfectants were then selected and maintained in DMEM-F12 medium containing $500 \mu \mathrm{g} / \mathrm{ml}$ hygromycin B, plus $600 \mu \mathrm{g} / \mathrm{ml}$ Zeocin (G418; Invitrogen Corp.). The expression of GFP-AFAP-110 protein was assessed by Western blotting, and, additionally, GFP-positive cells were visualized under a fluorescence microscope.

Immunoblotting. Immunoblotting was performed as described previously (41). Briefly, cells in log growth phase at $70 \%$ confluence were rinsed twice with ice-cold PBS and then lysed with RIPA A lysis buffer $(20 \mathrm{mM}$ sodium phosphate, $150 \mathrm{mM} \mathrm{NaCl}, 5 \mathrm{mM}$ sodium pyrophosphate, $5 \mathrm{mM}$ EDTA, $1 \%$ Triton X-100, $0.5 \%$ sodium deoxycholate, $0.1 \%$ SDS) supplemented with 1 tablet of complete mini-EDTA protease inhibitor cocktail (Roche Diagnostics) and $1 \mathrm{mM}$ sodium orthovanadate ( $\mathrm{pH}$ 7.4). Cells were harvested with the aid of a rubber policeman, clarified by centrifugation at $15,000 \mathrm{~g}$ for 15 minutes at $4{ }^{\circ} \mathrm{C}$, and prepared for Western blotting analysis. Lysates of the normal human prostatic epithelial cell line RWPE-1 were prepared as in previous reports (27). Total proteins ( $50 \mu \mathrm{g}$ from clarified cell lysates) were separated via $8 \%$ SDS-PAGE and electroblotted onto polyvinylidene difluoride membranes (Amersham Biosciences). The membranes were blocked with Tris-buffered saline with Tween $(0.15 \%)$ plus $5 \%$ dried nonfat milk for 30 minutes at room temperature and probed with the desired primary antibody diluted 1:1,000 in blocking buffer overnight at $4{ }^{\circ} \mathrm{C}$. Membranes were probed with antibodies to AFAP-110 (BD Biosciences), integrin $\beta 1$ (BD Biosciences), E-cadherin (Cell Signaling Technology), FAK (BD Biosciences), paxillin (Cell Signaling Technology), phospho-FAK 861 (BD Biosciences), phospho-paxillin 118 (Cell Signaling Technology), phospho-Src416 (Cell Signaling Technology), Src (Oncogene Science), paxillin (BD Biosciences), vinculin (Sigma-Aldrich), and $\beta$-actin (SigmaAldrich). Primary antibody incubation was followed by incubation with an HRP-conjugated secondary antibody (goat anti-mouse, sheep anti-rabbit; Bio-Rad) diluted 1:3,000 in blocking buffer for 1 hour at room temperature. Proteins were visualized with electrochemiluminescence detection reagents (PerkinElmer) followed by autoradiography.

Quantitation of autoradiograms. Autoradiographs were quantified as described previously (42) with Scion Image software program. The film was scanned using an HP Scanjet scanner. For each sample, the ratio of the density of the area of the sample to that of the corresponding area of actin was calculated.

Proliferation assays. PC3 cells and stable clones were plated in 12-well tissue plates in triplicate at 20,000 cells per well and returned to the incubator. Numbers of viable cells, determined by trypan blue exclusion, were counted with the aid of a hemacytometer under a Nikon ECLIPSE TE300 microscope. Cells were counted daily, until cultures reached saturation. The doubling time of each group of cells was calculated at the log phase of the corresponding proliferation curve using Microsoft Excel (version 2003).

Agarose colony-forming assays. Cells were suspended in $1 \mathrm{ml}$ of $10 \%$ FBS DMEM medium containing a $0.3 \%$ agarose and plated in triplicate on a firm $0.6 \%$ agarose base in 6 -well plates $(3,000$ cells/well) as described previously (43). Cells were then placed in a $37^{\circ} \mathrm{C}$ and $5 \% \mathrm{CO}_{2}$ incubator. Colonies of cells were allowed to grow over the course of 2 weeks. Images were obtained using a video camera (Optronics) mounted to a microscope (Leica DMIL). Then, $150 \mu \mathrm{l}$ of methylthiazole tetrazolium (MTT) was added to each well, and plates were returned to the incubator for 4 hours. Colonies with a diameter greater than $20 \mu \mathrm{m}$ were counted under a microscope (Nikon ECLIPSE TE300). The colony formation assay was performed in triplicate.

Migration assays. The modified Boyden chamber migration assay was used as described previously with minor modifications (44). PC3 cells or stable clones $\left(2.0 \times 10^{4}\right.$ cells $)$ were suspended in the upper well of the migration chamber (control inserts, $8 \mu \mathrm{m}$ pore size; $\mathrm{BD}$ ) in triplicate in $0.5 \mathrm{ml}$ of serum-free DMEM medium. The lower chamber was filled with $0.75 \mathrm{ml}$ of DMEM medium with $1 \%$ FBS. After 24 hours of incubation in a tissue culture incubator, the nonmigratory cells on the upper filter surface were removed with a cotton swab, and cells that had migrated to the bottom side of the insert were fixed and stained with Hema-3 (Biochemical Sciences) according to the manufacturer's instructions. The migratory cells were counted under a microscope at $\times 100$ magnification. Cell images were obtained using a video camera (Sony DXC-990) mounted to a microscope (Nikon Microphot-FX). Cells were counted in 5 random fields per insert.

Adhesion assays. PC 3 cells or stable clones were suspended in serum-free DMEM medium containing $1 \%$ BSA and plated in triplicate onto 96 -well plates $\left(1 \times 10^{5}\right.$ cells/well $)$, coated with laminin $(10 \mu \mathrm{g} / \mathrm{ml})$ or collagen $(10 \mu \mathrm{g} / \mathrm{ml})$. After incubation in a tissue culture incubator for 1 hour, cells were washed 3 times with PBS, while the input control groups were not washed. Cells were collected to the bottom of the plates by centrifuging at $150 \mathrm{~g}$ in a centrifuge with an adaptor for 96-well plates (IEC Centra-7R; International Equipment 
Company) at room temperature. The cells were then quantified by absorbance readings (optical density of $570 \mathrm{~nm}$ ), after MTT staining. The percentage of attached cells was obtained by dividing the optical density values of PBS-washed group with that of the respective input control group.

Immunofluorescence staining. Immunofluorescence staining was performed as described previously (45). Briefly, 5,000 cells in $0.2 \mathrm{ml}$ of DMEM medium with $10 \%$ FBS were plated per chamber in an 8-chamber slide. After 24 hours in an incubator, cells were fixed with $3.7 \%$ formaldehyde in PBS, permeabilized in PBS containing $0.5 \%$ Triton X-100 and 1\% BSA, and blocked with $10 \%$ FBS in PBS. Cells were incubated with an anti-vinculin (Sigma-Aldrich) or an anti-integrin $\beta 1$ (BD Biosciences) antibody diluted at 1: 50 in blocking solution. Cells were washed and incubated with Alexa Fluor 488- or Alexa Fluor 594-conjugated (green and red, respectively) anti-mouse or anti-rabbit IgG antibody (Jackson ImmunoResearch Laboratories Inc.) diluted at 1:100 in blocking buffer. Nuclei were stained with Hoechst 33342 (Molecular Probes; Invitrogen) at $1 \mathrm{mg} / \mathrm{ml}$. Slides were mounted with mounting medium (20 mM Tris, pH 8.0, 0.5\% $N$-propyl gallate, $90 \%$ glycerol) and examined with an epifluorescence microscope equipped with narrow-band pass excitation filters (Chroma) to individually select for green, red, and blue fluorescence. Cells were observed under a Hamamatsu C5810 camera mounted on a microscope (Nikon Microphot-FXA), and images were captured using Optimas image analysis software (Media Cybernetics).

Animals and orthotopic prostatic injections. Male athymic nude mice (NCI$n u / n u)$ were purchased from the Animal Production Area of the National Cancer Institute-Frederick Cancer Research Facility. The mice were housed and maintained under specific pathogen-free conditions and used for experiments when they were 6-8 weeks of age. All animal studies were performed in compliance with the regulations of the MD Anderson Cancer Center Animal Use and Care Committee.

For injection into the prostates of the nude mice, cells were harvested at $70 \%-80 \%$ confluence by rinsing with PBS followed by trypsinization with $1 \%$ trypsin. Viable cell number was counted by trypan blue exclusion with the aid of a hemacytometer after cells were suspended in DMEM F-12 medium with $10 \%$ FBS. After 1 wash with PBS, cells were resuspended in $\mathrm{Ca}^{2+}$ - and $\mathrm{Mg}^{2+}$-free HBSS and kept on ice. Intraprostatic injections were done as described previously (46). Briefly, male athymic nude mice were anesthetized with $0.5 \mathrm{mg} / \mathrm{g}$ body weight Nembutal (pentobarbital sodium; Abbott) injected into the peritoneal cavity (i.p.). A low midline incision was made through the skin and peritoneum. The urinary bladder and seminal vesicles were exposed and carefully pushed downward until the 2 lobes of the dorsal prostate could be seen. Tumor cells $\left(1.0 \times 10^{5}\right)$ in HBSS were injected into one of the exposed prostate lobes of the mice in a volume of $50 \mu \mathrm{l}$. The wounds were then closed using surgical steel wound staples. The growth of the tumors was monitored by external examination (palpation). The mice were sacrificed after 28 days. Tumors were excised, and tumor volume and weight were measured and recorded. Five mice were used for each experimental group.

Statistics. For quantification of AFAP-110 staining in the prostate tissue arrays, 2-tailed Student's $t$ test was used to compare the immunostaining scores of normal and BPH samples; normal and tumor samples; and samples from tumors with Gleason scores of at least 7 and those from tumors with Gleason scores of less than 7. For the analysis of the association between AFAP-110 expression levels and Gleason scores, prostate carcinoma samples with both low Gleason grade $(<7)$ and high Gleason grade $(27)$ were further divided into a low AFAP-110 expression group (negative and moderate staining with an immunostaining score less than 1.0) and a high AFAP-110 expression group (moderate and strong staining with an immunostaining score more than 1.0). The percentages of specimen numbers of each subgroup over total sample numbers were calculated and used to create a $2 \times 2$ cross table (Gleason score $<7$ or $\geq 7$ versus immunostaining score $<1.0$ or $\geq 1.0$ ). The Pear$\operatorname{son} \chi^{2}$ test was used to assess the correlation (47) of the low and high Gleason grades of tumor samples with low and high AFAP-110 expression.

For in vitro experiments, data are presented as mean and 95\% CI. Statistical analyses were performed with the 2 -sided Student's $t$ test where appropriate. For in vivo tumor growth, differences in tumor weights were determined using the Mann-Whitney $U$ test. A $P$ value of less than 0.05 was considered significant. SPSS software was used for all statistical analyses.

\section{Acknowledgments}

This work was supported by the US Department of Defense grant PC020017 and the Gillson Longenbaugh Foundation (to G.E. Gallick). J. Zhang is supported by the Sowell-Huggins Foundation. We thank Nila U. Parikh and Marjorie Johnson for excellent assistance with experimental techniques, and Michael J. Gray for helpful suggestions in the preparation of the siRNA constructs.

Received for publication October 23, 2006, and accepted in revised form July 9, 2007.

Address correspondence to: Gary E. Gallick, Department of Cancer Biology, Unit 173, The University of Texas MD Anderson Cancer Center, 1515 Holcombe Boulevard, Houston, Texas 77030, USA. Phone: (713) 563-4919; Fax: (713) 563-5489; E-mail: ggallick@mdanderson.org.
1. Kanner, S.B., Reynolds, A.B., Vines, R.R., and Parsons, J.T. 1990. Monoclonal antibodies to individual tyrosine-phosphorylated protein substrates of oncogene-encoded tyrosine kinases. Proc. Natl. Acad. Sci.U. S. A. 87:3328-3332.

2. Flynn, D.C., Leu, T.H., Reynolds, A.B., and Parsons, J.T. 1993. Identification and sequence analysis of cDNAs encoding a 110-kilodalton actin filament-associated pp60src substrate. Mol. Cell. Biol. 13:7892-7900.

3. Baisden, J.M., Gatesman, A.S., Cherezova, L., Jiang, B.H., and Flynn, D.C. 2001. The intrinsic ability of AFAP-110 to alter actin filament integrity is linked with its ability to also activate cellular tyrosine kinases. Oncogene. 20:6607-6616.

4. Qian, Y., Baisden, J.M., Zot, H.G., Van Winkle, W.B., and Flynn, D.C. 2000. The carboxy terminus of AFAP-110 modulates direct interactions with actin filaments and regulates its ability to alter actin filament integrity and induce lamellipodia formation. Exp. Cell Res. 255:102-113.

5. Qian, Y., et al. 2004. Analysis of the role of the leu- cine zipper motif in regulating the ability of AFAP110 to alter actin filament integrity. J. Cell. Biochem. 91:602-620.

6. Baisden, J.M., Qian, Y., Zot, H.M., and Flynn, D.C. 2001. The actin filament-associated protein AFAP-110 is an adaptor protein that modulates changes in actin filament integrity. Oncogene. 20:6435-6447.

7. Qian, Y., et al. 2002. PC phosphorylation increases the ability of AFAP-110 to cross-link actin filaments. Mol. Biol. Cell. 13:2311-2322.

8. Guappone, A.C., and Flynn, D.C. 1997. The integrity of the $\mathrm{SH} 3$ binding motif of AFAP-110 is required to facilitate tyrosine phosphorylation by, and stable complex formation with, Src. Mol. Cell Biochem. 175:243-252.

9. Haier, J., and Nicolson, G.L. 1999. Role of the cytoskeleton in adhesion stabilization of human colorectal carcinoma cells to extracellular matrix components under dynamic conditions of laminar flow. Clin. Exp. Metastasis. 17:713-721.

10. Defilippi, P., et al. 1999. Actin cytoskeleton organi- zation in response to integrin-mediated adhesion. Microsc. Res. Tech. 47:67-78.

11. Yuan, B.Z., Zhou, X., Zimonjic, D.B., Durkin, M.E., and Popescu, N.C. 2003. Amplification and overexpression of the EMS 1 oncogene, a possible prognostic marker, in human hepatocellular carcinoma. J. Mol.Diagn. 5:48-53.

12. Ormandy, C.J., Musgrove, E.A., Hui, R., Daly, R.J., and Sutherland, R.L. 2003. Cyclin D1, EMS1 and $11 \mathrm{q} 13$ amplification in breast cancer. Breast Cancer Res. Treat. 78:323-335.

13. Rodrigo, J.P., Garcia, L.A., Ramos, S., Lazo, P.S., and Suarez, C. 2000. EMS1 gene amplification correlates with poor prognosis in squamous cell carcinomas of the head and neck. Clin. Cancer Res. 6:3177-3182

14. van Rossum, A.G., Moolenaar, W.H., and Schuuring, E. 2006. Cortactin affects cell migration by regulating intercellular adhesion and cell spreading. Exp. Cell Res. 312:1658-1670.

15. Timpson, P., Lynch, D.K., Schramek, D., Walker, F., and Daly, R.J. 2005. Cortactin overexpression 
inhibits ligand-induced down-regulation of the epidermal growth factor receptor. Cancer Res. 65:3273-3280.

16. Gatesman, A., Walker, V.G., Baisden, J.M., Weed, S.A., and Flynn, D.C. 2004. Protein kinase Calpha activates c-Src and induces podosome formation via AFAP-110. Mol. Cell. Biol. 24:7578-7597.

17. O’Brian, C.A. 1998. Protein kinase C-alpha: a novel target for the therapy of androgen-independent prostate cancer? (Review-hypothesis). Oncol. Rep. 5:305-309.

18. Summy, J.M., and Gallick, G.E. 2003. Src family kinases in tumor progression and metastasis. Cancer Metastasis Rev. 22:337-358.

19. Koren, R., et al. 2004. Expression of protein kinase $\mathrm{C}$ isoenzymes in benign hyperplasia and carcinoma of prostate. Oncol. Rep. 11:321-326.

20. Migliaccio, A., et al. 2000. Steroid-induced androgen receptor-oestradiol receptor beta-Src complex triggers prostate cancer cell proliferation. EMBOJ. 19:5406-5417.

21. Amorino, G.P., Deeble, P.D., and Parsons, S.J. 2007. Neurotensin stimulates mitogenesis of prostate cancer cells through a novel c-Src/Stat $5 \mathrm{~b}$ pathway. Oncogene. 26:745-756.

22. Collins, A.T., and Maitland, N.J. 2006. Prostate cancer stem cells. Eur. J. Cancer. 42:1213-1218.

23. van Leenders, G.J., et al. 2003. Intermediate cells in human prostate epithelium are enriched in proliferative inflammatory atrophy. Am. J. Pathol. 162:1529-1537.

24. Feldman, B.J., and Feldman, D. 2001. The development of androgen-independent prostate cancer. Nat. Rev. Cancer. 1:34-45.

25. Maitland, N.J., and Collins, A. 2005. A tumour stem cell hypothesis for the origins of prostate cancer. BJU Int. 96:1219-1223.

26. Hudson, D.L., O'Hare, M., Watt, F.M., and Masters, J.R. 2000. Proliferative heterogeneity in the human prostate: evidence for epithelial stem cells. $L a b$. Invest. 80:1243-1250.
27. Wang, X., Clubbs, E.A., and Bomser, J.A. 2006. Genistein modulates prostate epithelial cell proliferation via estrogen- and extracellular signal-regulated kinase-dependent pathways. J. Nutr. Biochem. 17:204-210.

28. Reddig, P., and Juliano, R. 2005. Cancer Metastasis Rev. 24:425-439.

29. Carragher, N.O., and Frame, M.C. 2004. Focal adhesion and actin dynamics: a place where kinases and proteases meet to promote invasion. Trends Cell Biol. 14:241-249.

30. Adams, J.C. 2002. Regulation of protrusive and contractile cell-matrix contacts. J. Cell Sci. 115:257-265.

31. Brakebusch, C., and Fassler, R. 2005. $\beta 1$ integrin function in vivo: adhesion, migration and more. Cancer Metastasis Rev. 24:403-411.

32. Witkowski, C.M., Rabinovitz, I., Nagle, R.B., Affinito, K.S., and Cress, A.E. 1993. Characterization of integrin subunits, cellular adhesion and tumorgenicity of four human prostate cell lines. J. Cancer Res. Clin. Oncol. 119:637-644.

33. Dorfleutner, A., Stehlik, C., Zhang, J., Gallick, G.E., and Flynn, D.C. 2007. AFAP-110 is required for actin stress fiber formation and cell adhesion in MDAMB-231 breast cancer cells. J. Cell Physiol. In press.

34. Stewart, J.R., and O'Brian, C.A. 2005. Protein kinase C-alpha mediates epidermal growth factor receptor transactivation in human prostate cancer cells. Mol. Cancer Ther. 4:726-732.

35. Schaller, M.D., et al. 1992. pp125FAK a structurally distinctive protein-tyrosine kinase associated with focal adhesions. Proc. Natl. Acad. Sci. U. S. A. 89:5192-5196.

36. Turner, C.E. 2000. Paxillin interactions. J. Cell Sci. 113:4139-4140.

37. Ivaska, J., et al. 2003. Integrin-protein kinase C relationships. Biochem. Soc. Trans. 31:90-93.

38. $\mathrm{Ng}$, T., et al. 2001. Ezrin is a downstream effector of trafficking PKC-integrin complexes involved in the control of cell motility. EMBO J. 20:2723-2741.
39. Trevino, J.G., et al. 2006. Inhibition of SRC expression and activity inhibits tumor progression and metastasis of human pancreatic adenocarcinoma cells in an orthotopic nude mouse model. Am.J. Pathol. 168:962-972.

40. Trevino, J.G., et al. 2006. Src activation of Stat 3 is an independent requirement from NF-kappaB activation for constitutive IL-8 expression in human pancreatic adenocarcinoma cells. Angiogenesis. 9:101-110.

41. Summy, J.M., Trevino, J.G., Baker, C.H., and Gallick, G.E. 2005. c-Src regulates constitutive and EGFmediated VEGF expression in pancreatic tumor cells through activation of phosphatidyl inositol-3 kinase and p38 MAPK. Pancreas. 31:263-274.

42. Herynk, M.H., et al. 2003. Down-regulation of c-Met inhibits growth in the liver of human colorectal carcinoma cells. Cancer Res. 63:2990-2996.

43. Kim, S.J., et al. 2003. Reduced c-Met expression by an adenovirus expressing a c-Met ribozyme inhibits tumorigenic growth and lymph node metastases of PC3-LN4 prostate tumor cells in an orthotopic nude mouse model. Clin. Cancer Res. 9:5161-5170.

44. Minard, M.E., Herynk, M.H., Collard, J.G., and Gallick, G.E. 2005. The guanine nucleotide exchange factor Tiam 1 increases colon carcinoma growth at metastatic sites in an orthotopic nude mouse model. Oncogene. 24:2568-2573.

45. Gray, M.J., et al. 2005. HIF-1alpha, STAT3, CBP/ p300 and Ref-1/APE are components of a transcriptional complex that regulates Src-dependent hypoxia-induced expression of VEGF in pancreatic and prostate carcinomas. Oncogene. 24:3110-3120.

46. Davies, M.A., et al. 2002. Adenoviral-mediated expression of MMAC/PTEN inhibits proliferation and metastasis of human prostate cancer cells. Clin. Cancer Res. 8:1904-1914.

47. Paul, J.C., et al. 2005. MUC1, MUC2, MUC4, MUC5AC and MUC6 expression in the progression of prostate cancer. Clin. Exp. Metastasis. 22:565-573. 